\title{
En torno a la Onomástica Industrial en el español de Lima
}

\author{
Augusto Alcocer Martínez \\ Departamento Académico de Lingüística
}

5 obre el título del presente trabajo los especialistas han intentado varie(3) dad de términos: Julio Casares (1959: 282-285) la denominó "tecnicismo de las industrias"; Gunther Haensch et al. (1982: 416) acuñó el nombre "palabra marca"; el DRAE (1992) "marca registrada o nombre comercial". La francesa Jossete Rey-Devobe (1971: 88, 89) habla de "noms de marques" para el brasileño R.F. Mansur Guérios (1971) en cambio se trata de "oniônimos". En el Perú, Martha Hildebrandt (1998: 156) utiliza el rótulo de "marcas de fábrica" y finalmente Miguel Ángel Ugarte (1997) opta por "palabras comerciales".

El trabajo se hapropiestos ca de Letras

a) Recopilar materiales lingǘsticos de uso peruano (fundamentalmente de Lima) en la gran prensa limeña, en la narrativa literaria y en las Páginas Amarillas de la Guía Telefónica.

b) Señalar las peculiaridades de su empleo tanto en la comunicación oral como en la escrita.

c) Formación y productividad de esta Onomástica.

d) Contribuir al establecimiento de una caracterización del diasistema peruano. 
Se advierte que el presente estudio no es una tradicional lista de palabras ordenadas alfabéticamente; no es en sentido estricto una terminología; por último, tampoco se detiene en un asunto que toca nuestra identidad y a la vez resulta de enorme importancia para nuestra economía: La denominación de origen de productos ancestralmente peruanos.

\section{BREVE ANÁLISIS DE LA ESTRUCTURACIÓN DEL SIGNIFICADO}

Entre la variedad de modelos existentes para el análisis de la estructuración del significado elegiremos por adecuada la Teoría de los Campos Semasiológicos y C. Onomasiológicos (Kurt Baldinger. 1970)ํ․․

La Onomasiología representa la fase de las designaciones, al modo que la Semasiología encarna la fase de las significaciones. Un campo onomasiológico abarca todos los significantes: designaciones, nombres, etc. de un significado dado (según el Diccionario de F.C. Sáinz de Robles "delicado" se representa con cincuenta nombres $)^{2}$.

$\mathrm{Al}$ contrario, un campo semasiológico comprende todos los significados posibles que pueda contener un determinado significante (el mismo Sainz de Robles demuestra que "pie" cuenta con veintiocho significaciones diferentes). De lo expuesto, se desprehla que la Onomasiologia g.Ia Semasiología constituyen dos tipos de enfoque depfenómeriọ léxię-semántico, opuestos y a la vez complementarios.

Como apunta K. Baldinger, según la Teoría de la Comunicación: "la Onomasiología divisa los problemas desde la posición del que habla, de aquel que debe escoger entre diferentes medios de expresión (¿Cómo se llama...?). La Semasiología enfoca los problemas desde las perspectiva del que oye, del interlocutor que debe determinar la significación de la palabra que él entiende dentro de todas las significaciones posibles (¿Qué significa...?)

1 Kurt Baldinger (1970). Teoría semántica. Aguilar. Madrid.

2 Federico Sáinz de Robles (1981). Diccionario español de sinónimos y antónimos. Aguilar. Madrid. 


\section{LA ONOMÁSTICA INDUSTRIAL}

Perfilado grosso modo el sustento doctrinario en las líneas anteriores se hace necesario saber que se entiende por Onomástica, ciencia que ha visto considerablemente ampliado su campo de estudio ${ }^{3}$ en la Rusia actual, al conjunto de nombres propios de una lengua. Por considerarlo pertinente el autor se reducirá a tratar sólo dos aspectos clásicos de la disciplina, primero: La Antroponimia que se ocupa de los sustantivos propios que en una comunidad se aplican a los individuos integrantes, para distinguirlos unos de otros. Generalmente una persona se identifica mediante dos o más vocablos que forman una locución, el nombre de pila o nombre propio y el apellido. También se consideran los patronímicos, los apodos, el pseudónimo, el nombre clandestino, el sobrenombre, etc.; el segundo, la Toponimia cubre los nombres propios de lugares o accidentes geográficos, su estudio ha sufrido una alza en el Perú; los topónimos, refractarios a las alteraciones fonéticas, se mantienen sin mayores cambios, por esta razón son los que mejor testimonian las influencias extrañas que puedan ocurrir en una lengua.

En conclusión, para llegar a una definición del concepto me auxiliaré del autor Mansur Guerios (1971: 181) y deberá entenderse "como el nombre propio de producto comercial o industrial"; en otras palabras, en el contexto o frase lo que distinguirá un nombre de otro es que se debe encarar el nombre industrial como adjetivo, un calificativo que se opone al nombre común (sustantivo o locución sustantiva) parâ difereneiar necesariamente un producto de otro, de otra u otras fábrięs:-(Losiejemplos'sbar'riestros):

\section{lápiz Faber \\ lápiz Mongol \\ Jean Levis \\ Jean Kansas}

Antes de pasar a la presentación y análisis del corpus referido a la Onomástica Industrial en el Perú y siguiendo los lineamientos de Mansur Guerios "Oniônimia ou Onomástica Industrial" (1971) se proponen dos as-

3 N.V. Podolskaia (1978). Slovar russkoi onomasticheskoi terminologuii. Nauka. Moscú.

4 R.F. Mansur Guerios (1971). “Onionímia ou Onomástica Industrial”. En Estudos en Homenagen a Cándido Jucá (filho). Organizaçâo Simôes. Rio de Janeiro. 
pectos de estudio, uno aplicado a la Gramática de los nombres de fábrica y otro, a la Estilística de los nombres de marca (Dentro de la Afectividad del Lenguaje: emoción o sugestión en la creación y elección).

Se debe declarar que el modelo de análisis no es excluyente en el sentido de que una forma encerrada en la Gramática también podría encajar, con las explicaciones correspondientes, en el rubro de la Estilística:

memorex (Gramática, fonéticamente singular en el español por su terminación-x)

memorex (Estilística, el nombre se vincular significativamente con el objeto)

\section{GRAMÁTICA DE LOS NOMBRES INDUSTRIALES}

Aunque ciertas marcas de fábrica transgreden las normas de formación de las palabras castellanas, la mayoría de aquellas siempre se ajustan a los mecanismos morfológicos de la composición y la derivación.

\section{Ortografía}

Una mercancía puede lẹccibie infinidad \de nơmbres; unos agradables, ingeniosos; otros raros ojpoce agradables ató́do porgue al patrocinador del objeto sólo lo mueve el éxito de ventas en un mercado muy competitivo.

Iniciaremos el estudio observando palabras cuya escritura de letras es contraria a la índole del castellano ${ }^{5}$.

a) Con la letra -c, final de palabra:

Bic

Contac

Cognac

Klimatic (bolígrafo)

(fármaco)

(licor)

(electrodomésticos)

s Manuel Aragón P. (1988). Diccionario inverso de la lengua española. CONCYTEC. Lima. 
Pacific

Rayovac

(Tela de casimir)

(pilas)

b) $\mathrm{Con}-\mathrm{CH}$, inicial de palabra, con sonido $\mathrm{K}$ :

Cheracol

Chloromycetín

- $\mathrm{CH}$ - posición intermedia, con sonido $\mathrm{K}$ :

Bronchodermine

- $\mathrm{CH}$ africada, prepalatal, sorda:

Bosch

Crunch

Scotch

-CH-posición intermedia, africada, prepatal, sorda:

Anchor

c) Con -M, final de palabra:

Alivium

Denim

Ativam

Doloflam

Bactrim

Mentholatum

Diazepan/Valium

Nestum

e) Con - $T$, final de palabra:

Aldomet

Brut

Crest

\section{Eternit}

Nugget

Vitrovent

f) Con $-\mathrm{X}$, final de palabra

$\begin{array}{ll}\text { Ajax } & \text { Memorex } \\ \text { Apronax } & \text { Nordex } \\ \text { Betamax } & \text { Pirex } \\ \text { Coldex } & \text { Petromax } \\ \text { Kardex } & \text { Soldimix } \\ \text { Kleenex } & \text { Tampax } \\ \text { Lux } & \text { Xerox }\end{array}$


Entendidos como cambios de la forma de una palabra. La forma alterada por metaplasmo constituye una variante. En el ámbito de la diacronía, es la mudanza fonética de la palabra en su evolución. Los metaplasmos pueden ser de

Adición:

Comodoy (Comodo $+\mathrm{y}$ )

Cafetal (Café $+\mathrm{t}+\mathrm{al}$ )

Dunlopillo (Dunlop + illo)

Maicena (Maíz + ena)

Tubino (Tubo + ino)

O de Supresión:
Cerelac
(Cereal + lácteo)
Frigider
(Frigidaire)
Friol
(Freír + óleo)
Mapresa
(Madera + prensada + S.A.)
Nescafé
Nicovita
(Nestlé + café)
Petrolube
(Nicolini + vida)
Texaco
Vinifán
Vitrovent

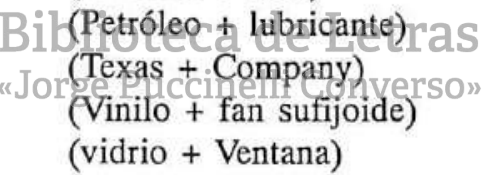

Morfología

Composición

Palabras-marcas simples:

Dralón

Colgate

Griffin

Karaoke

Lycra

Rexona

Yardley (fibra sintética)

(pasta de dientes)

(tinte para el calzado blanco)

(música de fondo que "ayuda" al cantante)

(fibra sintética)

(jabón)

(perfume) 
Locuciones/Compuestas:

a) Faber-Castell (lápices, bolígrafos) Mercedes Benz (vehículos)

b) Ambos elementos son sustantivos ligados por la preposición de: Emulsión de Scott (tónico)

Heno de Pravia (jabón)

Píldoras de Witt

c) El segundo elemento es una palabra que funciona como adjetivo:

Algodón Pima

Algodón Tangüis

Anís Nájar (aguardiente)

Caldos Maggie (saborizante de sobre)

Frotación Charcot

Jean Levys

Jabón Kauffman (ídem)

Leche Gloria (ídem)

Polvo Royal

Pollos Broaster

d) Uno de los elementos:es un yerbo: Letras Bebe crece (vestimenta para niños)

Rompeolla (marca de arroz)

e) Ambos elementos son sustantivos ligados por la conjunción y:

Concha y Toro (vino)

Rosas y Limón (jabón)

Derivación

a) Prefijación. Los prefijos de los nombres de fábrica son los mismos de los nombres comunes.

Hecta cloro

Monocrotofos

Microginón

Omnipén (plaguicida)

(plaguicida)

(anticonceptivo)

(fármaco) 
b) Sufijación

-al Mejoral, Doloral

-an Dristán

-il Aseptil, Cicatril, Clearsil, Dulcoryl, Sanaftil

-ol Adecerol, Cepacol, Cheracol, Epacol, Hisol, Pulmol

-ina Aspirina, Antalgina, Chiquitolina ("El Chavo del 8")

-san Dolorsán

Conservación del sufijoide derivativo

Si por voluntad intelectual o necesidad expresiva el inventor o fabricante de un producto industrial decide designarlo, por ejemplo, como:

Victrola Rayón o Toblerone (chocolate)

y el artículo tiene la fortuna de aleanzar rápido éxito de ventas es costumbre que luego concurran al mercado otros productos del mismo género y que adopten por razones obvias parte del nombre original.

En el caso del Castellano, la lengua ha adaptado a su propia índole fónico-gramatical nombres de fábrica que proceden del inglés u otras lenguas europeas.

\section{Biblioteca de Letras}

Un procedimiento muy socorindoles qa conservación del sufijoide derivativo:

-ola

Electrola

-on

gramola

radiola

rockola

Dralón

orlón

perlón -one

Winterone

De la marca registrada Celofán se desprende en el español limeño:

-fan

Vinifán

Atlasfán

(forro plástico)

Intifán

Pelifán

$\begin{array}{ll}\text { " } & " \\ \text { " } & "\end{array}$




\section{Sintaxis de Colocación}

Hay sintagmas y compuestos nacionales, o extranjeros cuyos elementos se hallan en orden directo y otros a la inversa:
a) Cinta Scotch
Jean Levis
Harina Blanca Flor
Vinagre Bully
b) Blue Jean
Cream Cracker
Walkman

\section{Extranjerismos}

\section{Del latín:}

Su existencia en nuestra variedad obedece a la innegable realidad de la dependencia económico-científica que sufre el Perú. A ello se suma la acción de los eficaces medios de comunicación masiva que alcanzan a todos los estratos sociales, desde los más rícos hasta los más desvalidos y que conforman la sociedad de consumo. En este contexto, los latinismos llegan a nuestras costas junto a las mercancías de Estados Unidos, Alemania, Japón, Inglaterra, Francia, Italia u otro país desarrollado. Veamos algunos casos:

Aqua Velva
Astra
Bacus
Capri
Faber
Factor
Forte
Gerovital
Lux
Nivea
Platinum Plus
Poroflex
Vestitex
Video
Vitaovo

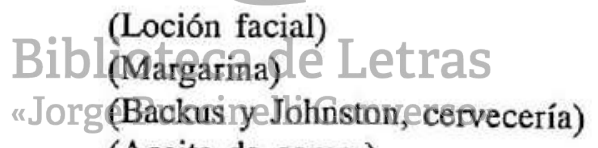

(Aceite de comer)

(Lápiz)

(Max Factor)

(Fortis, cerraduras)

(Rejuvenecedor)

(Jabón)

(Jabón)

Platinum Plus

(Hoja de afeitar)

Poroflex

(Espuma sintética)

(Industria del vestido)

(Técnica de grabar la imagen y el sonido)

Vitaovo

(Alimento para aves) 
Anglicismos:

Brasso

Eyemo

(Brass "metal", "latón")

Milkito

(Eye "ojo")

Vick Vaporub

(Yogurt, milk "leche")

(Ingl. "vapor" + rub "fricción")

Alemán:

Volkswagen

Wellapon

Wellazid

(Volks "pueblo", wagen "coche")

(Welle “onda", "ondulación”)

II. ESTILÍSTICA DE LA ONOMÁSTICA INDUSTRIAL

Designaciones del Industrial o Inventor

Los productos u objetos adquieren el apellido del fabricante o inventor.

Cardán

Carter

Chevrolet

Cinzano

Cretona

D'Onofrio

Faber

Max Factor

Ford

Guillete

Good Year

Levi Strauss

Listerine

Macádam

Pierre Cardin

Singer
(Gerolano Cardano). Pieza del automóvil.

(Carter). Parte del motor de-los automóviles.

(Louis Chevrolet). Fabricante de automóviles.

(Cinzano). "Licor dulce".

(Mr. Creton) Ciêrta tela de algodón.

(A. D'Onofrio), Chocolates y helados.

(Kasper Faber). Eápiz de nadera.

(Ídem). Fabricante de productós de belleza.

(Henry Ford). Fabricante de automóviles.

(King Camp Guillete). Hoja de afeitar.

(Charles Good Year). Descubridor del caucho vulcanizado y fabricante de neumáticos.

(Ídem). Pantalón deportivo de denim.

(Joseph Lister). Enjuague destinado a evitar el mal aliento.

(Mc Adam). Piedra triturada usada como base para una carretera.

(Ídem). Fabricante de ropa.

(Isaac Merrit Singer). Inventor-Modificador y fabricante de máquinas de coser. 
Siglificación
a) Siglas
DDT
TNT
CD
G.E.
LG
IBM
BMW
VHS
VW

b) Acrónimos

Reducción de una locución sustantiva a sus elementos, letras o sílabas, iniciales que forman en coljunto tm nombre propio:

ADIDAS

IMACO

LASER

NYLON

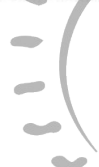

RADAR

\section{Biblioteca de Letras}

c) Terminados en $\longleftarrow$ TSA Puccinelli Converso»

COPSA

FAMESA

INRESA

LABRUSA

MAPRESA

VAINSA

Nombres de personajes célebres reales o ficticios:
Aladino
(Termos)
Bolívar
(Jabón de lavandería)
Goliat
(Bicicletas)
King Kong
(Ladrillos)
Sansón
(Recipiente enorme de material plástico) 
Nombres de Santos

La pureza espiritual de los santos se vincula a la pureza de las aguas.

Aguas minerales:

San Antonio

San Luis

San Cayetano

San Mateo

Nombres de Gentilicios:

Cerveza Arequipeña

Cerveza Cuzqueña

Chilcano (sopa)

Arroz Costeño
Plátano Maleño

Salteña (empanada)

Sampedrano (pellón)

Scotch (cinta pègante)

Denominación de Origen:

Nombre que se asigna a un producto determinado que es propio de una zona geográfica y que lleva el nombre de đicha área:

Caña de Guayaquil

Cemento Portland

Pino Oregón

Chancay (bizcocho)

Pisco (aguardiente de uva)

Habano (puro) Biblioteca d Biedra de Huamanga

Nombres cuyos sentidos se relacionan con la cosa:

$\begin{array}{ll}\begin{array}{l}\text { Cerelac } \\ \text { Chocopresto }\end{array} & \begin{array}{l}\text { (cereal }+ \text { leche) } \\ \text { (chocolate }+ \text { presto) } \\ \text { Clorina }\end{array} \\ \text { Clorox } & \text { (cloro) } \\ \text { Dolorsán } & \text { (cloro) } \\ \text { Dulcoryl } & \text { (dolor }+ \text { san) } \\ \text { Mejoral } & \text { (Mulce }+ \text { ryl) } \\ \text { Memorex } & \text { (Memora }+ \text { l) } \\ \text { Sanafitil } & \text { (Sana }+ \text { feet }+ \text { il) } \\ \text { Vitrovent } & \text { (vidrio + ventana) }\end{array}$


Nombre de letras y abreviaciones numéricas:

Aceite 3 en 1

Automóvil $0 \mathrm{~km}$.

Champú 2 en 1

Cinta $3 \mathrm{M}$

Muebles 501

Seven Up (7 up)

B12 (vitaminas)
Vino 4 Bocas

Triple Kola

Alberto VO5 (champú)

Bond A4

Parker 61

$20^{\text {th }}$ Century Fox

De palabra-marca a sustantivo común/Lexicalización de las palabras comerciales $^{6}$ :
Ajinomoto
Alka-seltzer
(Sazonador)
Baquelita
(Calmante)
Bayer
Betamax
(Resina sintética...)
Bividí
(Valles de Mala-Cañete, "insecticida")
(Videograbadora)
Brasso
(Ropa interior sin mangas, Huiri-huiri, Arequipa)
Broster
Caterpillar
(Abrillantador de metales)
(Cierta clase de pollo preparado)
Cuáquer
Chicle
Cinzano
Combi
(Vehículo oruga con una excavadora)
Los Comercios
Guillete
Maicena
Nescafé
(Avena)
Pírex
Primus
Sapolio
Termo
(Vidrio resistente al fuego)
(Cocina a kerosene)
(Cualquier jabón detergente)
(recipiente aislante)

- Héctor E. Solari (1962). "Lexicalización de marcas comeciales: presentación de algunos casos registrados en el habla rioplatense". En Boletín de filología. Montevideo. T. IX, pp. 41-55. 
Terokal

Teflón
(Pegamento industrial)

(antiadherente)

El nombre del fabricante aparece disimulado a combinación con el nombre de la cosa:
Magna pen
Nicovita
(Magma + penicilina)
Vencelatex
(Nicolini + vita)
(Vencedor + latex)

\section{Uso figurado}

Barbie. Muñeca. Con el significado de "cosa, objeto".

Miss Mundo 1996: “...he demostrado al mundo que una reina de belleza es ante todo una mujer que bien puede ganar como perder peso. Que se cansa; tiene ganas de comer y dormir como cualquiera, pero, sobretodo, que tiene opiniones propias. Aunque yo sé que ellos prefieren una Barbie yo no he dejado de ser Alicia Machado por ser Miss Universo, concluye".

En El Comercio de Lima 11/V/1997, p. C15.

Cinzano. Licor dulce. "Melancólico, tierno, acaramelado".

"Cuando llegó lạnōche y-se presentaron_los muchachos, tú y Mónica estaban tan solos y tan cinzano".

José Antonio Bravo. Barrio de broncas 1972, p. 178.

Chizito. Pasabocas de queso y maíz. "Pequeñez, modestia".

a) "No quiero que se suba el primer puesto a la cabeza y sentir que soy el último chizito del tono. El primer puesto es algo relativo, tuve más suerte, menos nervios, no sé" nos dice con humildad D.M.G. primer puesto en el examen de ingreso.

b) En el lenguaje de los jóvenes "miembro viril".

En El Comercio de Lima. 19/II/1995, p. G4. 
Coca Cola. Bebida gaseosa.

a) Juventud

b) Adj. Alocado, sobrado.

Erectol. Droga contra la disfunción eréctil masculina.

"Levantar".

"Proyectar una película para dos gatos no debe salir cuenta $[. .$.$] De otro$ modo el Cine Roma morirá y no habrá erectol que lo levante..."

En El Comercio de Lima 15/IX/1992.

Frigidaire. Marca de refrigeradora o nevera. "Depósito".

"El encanto del recuerdo reside en lo que fue no será jamás", acertó a escribir Federico More, acordándose de su futuro: haber sacado del "frigidaire" de los archivos sus crónicas gastronómicas y poder leerlas hoy en un libro...".

En El Comercio de Lima 20/1X/1998, p. A14.

Mejoral. Analgésico. "Ayuda, colaboración, aporte".

"Todo el mundo se ha olvidado de mí. Cuando estaba hospitalizado, mis amigos no me llevaron ni siquiera una 'mejoral' ".

En La República de Lima 8/VI/1984, p. 56.

Tampax. Toalla higiénica.

"Las historias de amor de floy son las det príncipe Carlos y Camila Parker-Bowles [...] de tođas fascanecdotas de estála relación la más famosa sigue siendo la conversación privada en la que Carlos le dice que le gustaría ser su Tampax".

En El Comercio de Lima 11/1I/2001, p. 4.

Teflón. Sustancia antiadherente "Escudo, barrera".

"El bajo perfil, el secreto, la discreción y la asepsia son parte del efecto teflón del fujimorismo ante la ciudadanía. Los medios son utilizados como la barrera de ruido público que asordina las debilidades humanas del personal fujimorista".

En La República de Lima 14/V/1985, p. 4.

Viagra. Fármaco contra la impotencia masculina. "Anciano, viejo". Al abuelo le dicen 'tío Viagra'. 
Volvo. Cierta marca de vehículos pesados. En el Callejón de Conchucos (Ancash) se aplica a toda persona gorda:

La volva Teresa.

Fraseologismos

Tomados en su mayoría de la industria del cine, son conjuntos expresivos casi fijos (locuciones, frases hechas, colocaciones habituales, etc.) conocidos y utilizados en nuestra comunidad:

Hacer algo Ben Hur (alargar un asunto)

Una caja de Leche Gloria (colocación hab., de sentido patente).

La corneta de D'Onofrio (colocación hab., llamada de atención del helade-

La Dolce Vita ro o chocolatero de la marca)

Suspenso a lo Hitchcock (en ascuas, pendiente, perplejidad)

- Ser uno como el León de la Metro

(aplícase a la persona que "ruge" pero no hace đaño)

El Llanero Solitario (individual, personal, referido al trabajo y actuación)

El perrito de la Vícton ibl (expresión de sentido literal)

Rebelde sin Causa (colocación, desđe los anos 60 hasta el presente,

En tecnicolor

Yon Huaino joven contestatario y que practica la libertad)

(a colores)

(humorístico, John Wayne)

\section{DIARIOS CONSULTADOS}

El Comercio de Lima, desde 1990 hasta el 2001

La República de Lima, desde 1990 hasta el 2001. 